\title{
Novel Supervisory Technique Using Wavelength-Division-Multiplexed OTDR in EDFA Repeatered Transmission Systems
}

\author{
Y. W. Lai, Y. K. Chen and W. I. Way, Senior Member, IEEE
}

\begin{abstract}
A wavelength-division-multiplexed opticaltime-domain-reflectometry (OTDR) supervisory technique is experimentally demonstrated in a $200-\mathbf{k m}$ transmission system. The technique can simultaneously achieve in-line EDFA performance monitoring and diversity protection, and fault location in optical fibers.
\end{abstract}

\section{INTRODUCTION}

$\mathbf{T}$ HE introduction of erbium-doped fiber amplifiers (EDFA's) in a long-distance transmission system requires a practical supervisory method to offer in-service monitoring and fault-locating capability. The supervisory method should be suitable not only for monitoring the status of EDFA's, but also for fault-locating fiber breaks in the transmission system.

There have been three types of supervisory techniques reported: (1) by modulating a pump laser diode with a multi$\mathrm{Mbit} / \mathrm{sec}$ signal [1] or with a several hundred bit/sec telemetry signal [2] in an EDFA, or by modulating the spontaneous emission in a semiconductor optical amplifier [3], (2) by using optical circulators to provide a reverse path for the amplified back-scattered OTDR [4] or for an amplified supervisory signal [5], and (3) by using "on-board" OTDR with pulsed pump lasers [6]. Method (1) can supervise the performance of each individual EDFA, but cannot locate a fiber cut in the system; method (2) requires a costly circulator and an additional optical fiber link [4], or additional fine-tuned DFB lasers [5]; Method (3) cannot utilize a conventional OTDR from a remote head-end.

In this paper, we propose and experimentally demonstrate a new supervisory method which uses a wavelength-divisionmultiplexed (WDMed) OTDR. The method requires only a minor modification of the convention OTDR technique, and can simultaneously monitor the performance of all inline EDFA's (which are equipped with optical isolators) and locate a fiber break in the system with acceptable precision. The experiment successfully tested a transmission system with three in-line EDFA's and a total fiber length of 200 km.

Manuscript received October 19, 1993; revised December 27, 1993. Y. W. Lai and Y. K. Chen are with the Institute of Electro-Optical Engineering, National Chiao-Tung University, Hsinchu, Taiwan, ROC.

W. I. Way is with the Department of Communication Engineering, National Chiao-Tung University, Hsinchu, Taiwan, ROC.

IEEE Log Number 9400071.

\section{OPERATION PRINCIPLE}

The operation principle is illustrated in Fig. 1. An OTDR operating at a wavelength $\lambda 1$ is combined with a transmitted signal at $\lambda 2$ by a WDM multiplexer. In order to monitor fiber cut and all "uni-directional" working EDFAs' status ("uni-directional" because of the included optical isolators), the OTDR wavelength is passed around each uni-directional working EDFA's by a pair of input/output WDMs. A bidirectional protection optical amplifier which amplifies the OTDR wavelength $\lambda 1$ in both directions can be inserted between the input and output WDMs, as shown in Fig. 1, to increase the OTDR dynamic range. The pump laser of a bi-directional protection amplifier is used under normal conditions, and is used to replace the failed pump laser in a working amplifier under failure conditions. A local monitoring control circuit is used to activate a mechanical optical switch so that the original failed working pump laser can be replaced. In the mean time, the OTDR at the transmitter end can observe a sharp drop on the OTDR trace due to a strong absorption in the unpumped protection EDFA. Therefore, both status monitoring and protection for the working EDFA can be achieved. If the transmission system is not long and a large OTDR dynamic range is not required, e.g., in a single inline amplifier transmission system, the bi-directional EDFA's can be replaced by a semiconductor optical amplifier (SOA) at $1.3 \mu \mathrm{m}$, or even by a simple mechanical optical switch. In the former case, when a working EDFA fails, the control electronics simply turns off the SOA so that a sharp sink appears on the head-end OTDR trace. In the latter case, a mechanical optical switch can be switched from close to open position so that a sharp spike appears on the head-end OTDR trace.

\section{EXPERIMENTS}

In our experiments, the $1.3 \mu \mathrm{m}$ and $1.55 \mu \mathrm{m}$ Fabry-Perot lasers in a commercial OTDR (HP8146A) were replaced by two DFB lasers at wavelengths $1.318 \mu \mathrm{m}$ and $1.556 \mu \mathrm{m}$, respectively. For a pulse period of $15 \mu \mathrm{s}$, the peak power of the probe pulse at the OTDR output single-mode fiber port was $-5.0 \mathrm{dBm}$ for $1.318 \mu \mathrm{m}$ operation and $-6.8 \mathrm{dBm}$ for $1.556 \mu \mathrm{m}$ operation. The feasibility of the proposed scheme was demonstrated using both bi-directional EDFA's at 1.556 $\mu \mathrm{m}$ and a bi-directional SOA at $1.318 \mu \mathrm{m}$. 


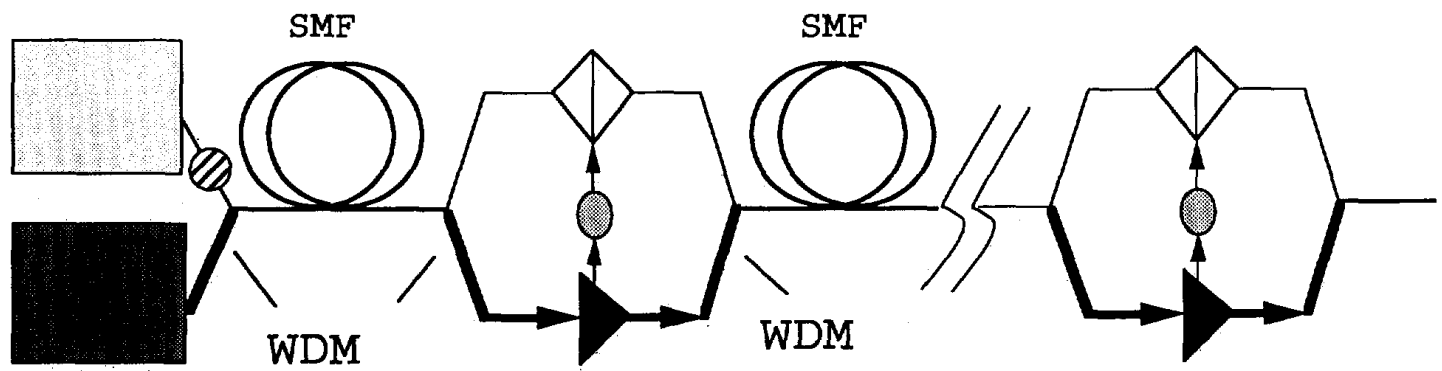

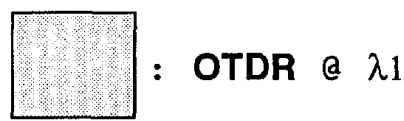

: Signal @ $\lambda 2$

\section{$\bigoplus$ : Optical Filter}

Fig. 1. Operational principle.

For the $1.556 \mu \mathrm{m}$ case, we tested the OTDR performance with two stages of bi-directional EDFA's with $75 \mathrm{~km}$ interspan, and with three stages of bi-directional EDFA's with $50 \mathrm{~km}$ interspan. All EDFA gains were adjusted to compensate for the interspan loss. Average gain per EDFA stage is about $16 \mathrm{~dB}$ for the $75-\mathrm{km}$ interspan case, and about $10 \mathrm{~dB}$ for the $50-\mathrm{km}$ interspan case. To reduce the effect of amplified spontaneous emission noise, an optical filter with a 3-dB bandwidth of $0.4 \mathrm{~nm}$ and an insertion loss of $0.5 \mathrm{~dB}$ was placed at the OTDR output port, as shown in Fig. 1. The observed OTDR traces for $15 \mu$ s pulses are shown in Fig. 2(a) and 2(b), respectively. Note that the signal wavelength can be anywhere between 1.530 to $1.560 \mu \mathrm{m}$, as long as it differs from the OTDR wavelength so that a WDM mux/demux pair with sufficient wavelength isolation and low back reflection is available. A WDM mux/demux pair was not actually used in the experiment. However, commercially available WDMs in the $1550 \mathrm{~nm}$ region exhibit more than $30 \mathrm{~dB}$ wavelength isolation and lower than $-40 \mathrm{~dB}$ back reflection, and should therefore be usable in the proposed system.

For the $1.318 \mu \mathrm{m}$ case, due to the relatively low gain and polarization-dependent characteristics of a commercial SOA, we tested with a single stage only. The SOA was biased to render a fiber-to-fiber TE gain of $16.8 \mathrm{~dB}$ (with TE and TM gain difference of about $8 \mathrm{~dB}$ ), and was inserted between two spools of single-mode fibers with a length of $75-\mathrm{km}$ and 50 $\mathrm{km}$, respectively. An optical filter with a 3-dB bandwidth of 1.6 $\mathrm{nm}$ and an insertion loss of $1.3 \mathrm{~dB}$ was placed at the OTDR output port. The measured result is shown in Fig. 3. Note that a common 1.3/1.55 $\mu \mathrm{m}$ WDM mux/demux pair can be used (typically with more than $40 \mathrm{~dB}$ wavelength isolation and lower than $-40 \mathrm{~dB}$ backreflection). The average gain offered by the SOA, as can be seen from Fig. 3, was about $12.6 \mathrm{~dB}$ which is the average between the forward TE gain and the backward polarization-dependent gain. Due to the existence

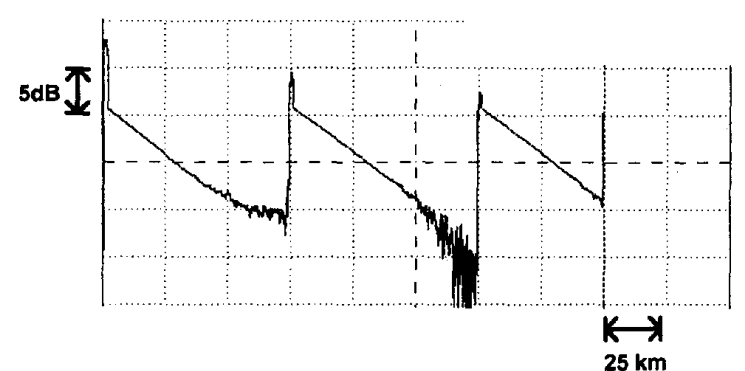

(a)

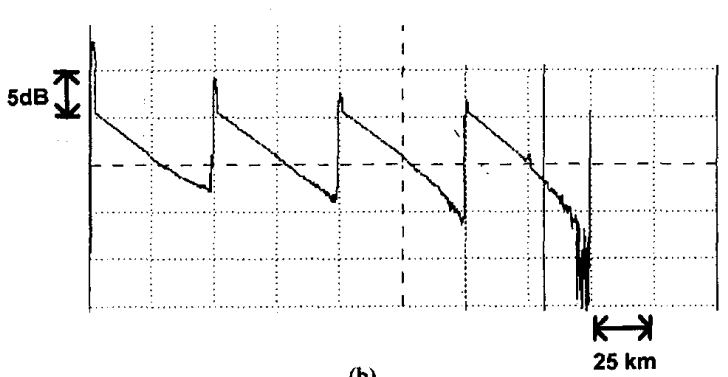

Fig. 2. $1.556 \mu \mathrm{m}$ OTDR via (a) three stages EDFA and four $50 \mathrm{kminterspan}$, (b) two stages of EDFA, two $75 \mathrm{~km}$ interspans, and a final $50 \mathrm{~km}$ span. The pulse width was $15 \mu \mathrm{s}$. Number of averaging $=1$.

of about $-30 \mathrm{~dB}$ reflection at both facets of an SOA, higher gain SOAs may cause multiple "echoes" in the OTDR trace for cascaded SOAs and create an illusion of more amplifiers than there actually are [7].

As mentioned earlier, commercial WDM devices for both $1.3 / 1.55 \mu \mathrm{m}$ and say, $1.53 / 1.55 \mu \mathrm{m}$, all have wavelength isolation better than about $30 \mathrm{~dB}$. This gives electrical interference of lower than $60 \mathrm{~dB}$ and should not have any effect on the signal channel. 


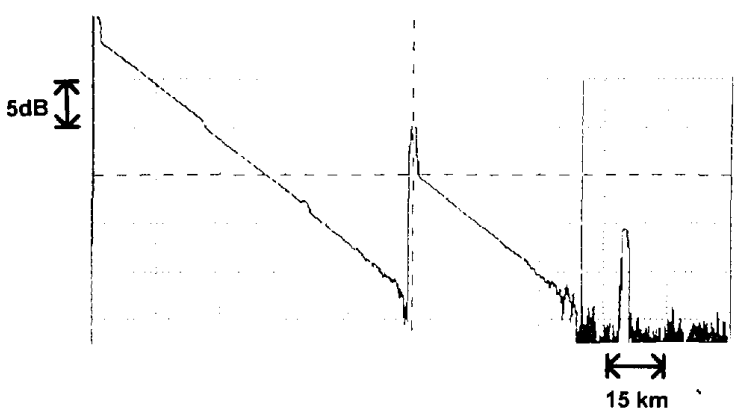

Fig. 3. $1.318 \mu \mathrm{m}$ OTDR via a single-stage SOA. The fiber lenghts before and after the SOA were $75 \mathrm{~km}$ and $50 \mathrm{~km}$, respectively. The pulse width was $15 \mu \mathrm{s}$. Number of averaging $=301$.

\section{SNR Calculation}

The received SNR of the backscattered light with in-line bi-directional EDFA's is calculated in this Section to give a guideline regarding the constraints of the proposed supervisory technique, i.e., how many cascaded EDFA's can be used and what is a better choice for the interstage span. The SNR is given as follows:

$$
\mathrm{SNR} \cong \frac{I_{r}^{2}}{N_{\text {shot }}+N_{\mathrm{sp}-\mathrm{sp}}+N_{\mathrm{th}}}
$$

where $I_{r}$ is the received photocurrent of the backscattered light and is given by

$$
I_{r}=\frac{S \alpha_{S} W v_{g}}{2} P_{\text {in }} \exp (-2 \alpha L) \cdot\left(\frac{q \eta}{E}\right) \cdot M
$$

where $S$ is the recapture coefficient, $\alpha_{S}$ (neper/m) is the Rayleigh scattering loss, $W$ is the pulse width of the OTDR probe pulse, $v_{g}$ is the group velocity of light in the fiber, $P_{\text {in }}$ is the peak power of the probe pulse output from OTDR equipment, $\alpha$ is the fiber loss, $L$ is the interstage span length, $q$ is the electron charge, $\eta$ is the quantum efficiency of the photodetector, $E$ is the photon energy and $M$ is the APD avalanche gain. The noise currents in eq. (1) include shot noise, spontaneous-spontaneous beat noise, and the receiver thermal noise. Note that the signal-spontaneous beat noise has been neglected because the backscattered signal power is much smaller than ASE power. The definitions of the first two noise currents in eq. (1) are given as follows:

$$
\begin{aligned}
N_{\text {shot }} & \cong 2 B_{e} \cdot q\left(I_{r}+I_{\mathrm{sp}}\right) M^{1+x} \\
N_{\mathrm{sp}-\mathrm{sp}} & =B_{e}\left(\frac{I_{\mathrm{sp}}^{2}}{B_{o}}\right)
\end{aligned}
$$

where $B_{e}$ is the electrical bandwidth of the OTDR receiver, $x$ is the excess noise factor, $B_{o}$ is the optical bandwidth of the optical filter shown in Fig. 1. The dark current is neglected in eq. (3). The ASE photocurrent at the OTDR receiver, is given by

$$
I_{\mathrm{sp}}=2 n_{\mathrm{sp}}(G-1) E B_{\mathrm{o}} N \exp (-\alpha L) \cdot\left(\frac{q \eta}{E}\right) \cdot M
$$

where $n_{\mathrm{sp}}$ is the EDFA population inversion parameter, $G$ and $N$ are the gain and number of the in-line amplifiers, respectively.

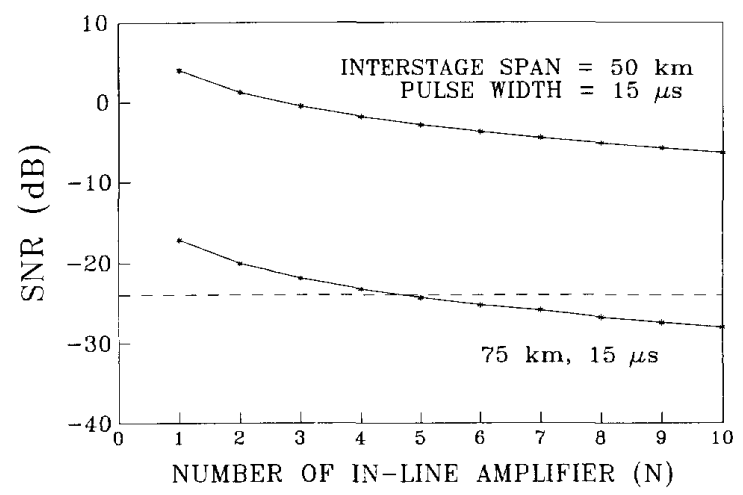

Fig. 4. Recieved SNR in OTDR versus number of in-line amplifiers. The dashed line represents the minimum recieved SNR which is required to achievea final SNR of unity after digital averaging improvement.

In our experiment, $B_{o}=50 \mathrm{GHZ}, B_{e}=400 \mathrm{kHz}$, $N_{\text {th }}=4 \times 10^{-19}\left(A^{2}\right), x=0.7, N_{\text {sp }}=2.5, M=20$, and, the responsivity of the APD is approximately $15 \mathrm{~A} / \mathrm{W}$. The EDFA gain were 10 and $16 \mathrm{~dB}$ for fiber spans of 50 and 75 $\mathrm{km}$, respectively. The OTDR pulse was $15 \mu \mathrm{s}$. Fig. 4 shows the calculation results based on the parameters given above, which gives the received SNR in the APD receiver versus the number of in-line amplifiers $N$. The SNR improvement of the HP8146A was estimated to be $23.5 \mathrm{~dB}$, therefore a minimum SNR should be about $-23.5 \mathrm{~dB}$ to achieve a final $\mathrm{SNR}=1$ after digital averaging improvement. We can see from Fig. 4 that for an interstage span of $50 \mathrm{~km}$, the OTDR supervisory technique can be applied to more than 130 stages of cascaded bi-directional EDFA's; for an interstage span of $75 \mathrm{~km}$, the OTDR supervisory technique can be applied to a link with only 4 stages of cascaded EDFA's. For an optimum interstage distance of $50 \mathrm{~km}$ which requires each EDFA gain to be about $10 \mathrm{~dB}$, the optical back reflection of $<-40 \mathrm{~dB}$ for WDM mux or demux should not cause any problem such as multiple echoes [7].

\section{CONCLUSION}

A novel supervision technique is proposed and experimentally demonstrated for lightwave transmission systems using in-line (uni-directional) EDFA's. The proposed technique, which requires only slight modification of the conventional OTDR method, can be used for simultaneously monitoring EDFA performance and locating a fiber cut between EDFA's. Furthermore, the pump laser used for a bi-directional OTDR amplifier can work also as a standby laser in case of the pump laser in the working EDFA fails. Based on the proposed WDMed OTDR principle, we have demonstrated a total distance up to $200 \mathrm{~km}$ for $1.55 \mu \mathrm{m}$ OTDR supervisory system, and up to $125 \mathrm{~km}$ for a $1.3 \mu \mathrm{m}$ OTDR supervisory system. A complete system analysis given in this paper provides a general guideline for choosing the appropriate interstage span in a long-distance link so that the OTDR supervisory technique can be used. 


\section{ACKNOWLEDGMENT}

This work was supported in part by National Science Council, Republic of China, under contract number NSC830417-E009-012. The authors would like to thank Mr. Frank Maier of Hewlett Packard at Boelingen Instrument Division, Germany, for providing and supporting the HP8146A OTDR. They would also like to thank Telecommunications Laboratories, Ministry of Communications, Taiwan, ROC, for loaning the multiple stages of EDFA.

\section{REFERENCES}

[1] S. Matsuoka, Y. Yamabayashi and K. Aida, "Supervisory signal transmission methods for optical amplifier repeater systems," Technical Digest, GLOBECOM'90, paper 903.2.
[2] M. Angellieri, et al, "Low bit-rate service channel for remote monitoring of EDFA in-line repeaters obtained by modulation of the active fiber gain," Technical Digest, Optical Amplifiers and Their Applications, 1992, Paper ThA3.

[3] A. D. Ellis, W. A. Stallard and D. J. Malyon, "Supervisory system for cascaded semiconductor laser amplifier repeaters," Electron. Lett., vol. 25, pp. 309-311, 1989.

[4] Y. Sato and K. Aoyama "Optical time domain reflectometry in optical transmission lines containing in-line Er-doped fiber amplifiers," $J$. Lightwave Technol., vol. 10, pp. 78-83, 1992.

[5] Y. Sato, Y. Yamabayashi and K. Aoyama, "Supervisory channel using fiber Brillouin amplifiers for Er-doped fiber amplifier transmission systems," Technical Digest, Optical Amplifiers and Their Applications, 1992, Paper ThA2.

[6] J. C. MacKichan, J. A. Kitchen and C. W. Pitt, "Innovative approach to interspan fiber break location in fiber amplifier repeatered communication systems," Electron. Lett., vol. 28, pp. 626-628, 1992.

[7] L. C. Blank and J. D. Cox, "Optical time domain reflectometry on optical amplifier systems," J. Lightwave Technol., vol. 7, pp. 1549-1555, 1989. 